\title{
Universal features of the Abelian Polyakov loop in $1+1$ dimensions
}

\author{
J. Unmuth-Yockey, ${ }^{1,}$ Jin Zhang, ${ }^{2, \dagger}$ A. Bazavov, ${ }^{3}$ Y. Meurice, ${ }^{4}$ and S.-W. Tsai ${ }^{2}$ \\ ${ }^{1}$ Department of Physics, Syracuse University, Syracuse, New York 13244, USA \\ ${ }^{2}$ Department of Physics and Astronomy, University of California, Riverside, California 92521, USA \\ ${ }^{3}$ Department of Computational Mathematics, Science and Engineering, \\ and Department of Physics and Astronomy, \\ Michigan State University, East Lansing, Michigan 48824, USA \\ ${ }^{4}$ Department of Physics and Astronomy, The University of Iowa, Iowa City, Iowa 52242, USA
}

(Received 8 August 2018; published 27 November 2018)

\begin{abstract}
We show that the Polyakov loop of the two-dimensional lattice Abelian Higgs model can be calculated using the tensor renormalization group approach. We check the accuracy of the results using standard Monte Carlo simulations and find good agreement. We show that the energy gap produced by the insertion of the Polyakov loop obeys universal finite-size scaling which persists in the continuous-time limit. We briefly discuss the relevance of these results for quantum simulations.
\end{abstract}

DOI: 10.1103/PhysRevD.98.094511

\section{INTRODUCTION}

Two-dimensional gauge models have played an important role in our understanding of four-dimensional quantum chromodynamics (QCD). They appear prominently in several of the Coleman's Erice lectures [1] and provide nontrivial model calculations for questions related to confinement, topology and symmetry breaking. For these reasons, they are often the first targets when new methods are developed. There has been a recent interest in using controlled quantum systems to perform calculations in lattice gauge theories. The methods used include cold atoms in optical lattices, trapped ions, and state of the art quantum computers. Recent efforts have been focused on the Schwinger model [2-5] and its scalar counterpart the two-dimensional Abelian Higgs model [6-8].

In recent years, the tensor renormalization group method (TRG) has been used to reformulate spin and gauge models with compact field variables into models of discrete integer (or half-integer) fields [9-13]. This reformulation uses discrete character expansions which are suitable for quantum computations and can also be used for sampling purposes [14]. The computation of the tensors involves integration over the field variables and is manifestly gauge invariant. The TRG has been used to reformulate the twodimensional Abelian Higgs model and find approximations

\footnotetext{
jufunmuth@syr.edu

jzhan039@ucr.edu
}

Published by the American Physical Society under the terms of the Creative Commons Attribution 4.0 International license. Further distribution of this work must maintain attribution to the author(s) and the published article's title, journal citation, and DOI. Funded by SCOAP ${ }^{3}$. suitable to implement the model on optical lattices [15]. Recently developed experimental methods involving Rydberg atoms [16] have been exploited to propose realistic implementations of the model on a physical ladder [17]. A key aspect of the proposal in Ref. [17] is that gauge invariance is maintained exactly, and the Gauss law constraint need not be enforced "by hand." This differs from other quantum simulation approaches $[3-8,18,19]$.

In order to test the ladder implementation, it was proposed to measure the Polyakov loop [17] for the two-dimensional Abelian Higgs model. In this process, we found remarkable finite-size scaling (FSS) properties that to the best of our knowledge have never been reported. In this article, we describe these calculations and the interpretation of the results.

The paper is structured as follows: In Sec. II, the reformulation of the model in terms of the discrete fieldquantum-number variables is briefly reviewed, and the Polyakov loop is introduced in terms of the reformulated variables. We emphasize that the Brout-Englert-Higgs mode is taken to be infinitely massive and that we are left with the compact Nambu-Goldstone modes and compact gauge fields. The model that we are considering could be called "compact scalar electrodynamics".

In Sec. III, numerical calculations in the relativistic Lagrangian formulation where space and Euclidean time are on the same footing are reported. We first show that the TRG method based on coarse graining and the standard Monte Carlo (MC) sampling on the original fields are in good numerical agreement. We then show that the Polyakov loop defines an energy gap, $\Delta E$, that can be extracted from lattice configurations with different temporal lengths. We report on the FSS of this energy gap and present results across a range of spatial sizes and gauge couplings. 
In Sec. IV, we review the continuous-time limit for this model. We then relate this continuous-time limit in the field-quantum-number representation to a Hamiltonian in the charge-variables representation. Next we derive the continuous-time limit of the Polaykov loop and show that its insertion can be realized by a local modification of the original Hamiltonian. It is here that we show that the FSS observed in the isotropic coupling case survives the continuous-time limit of this model and exhibits similar data collapse. Finally, we consider the limit of zero gauge coupling where the model reduces to that of the $\mathrm{O}(2)$ spin model, and we give justification for the accuracy of our results.

In Sec. V, we relate a special choice of boundary conditions in this model to the inclusion of the Polyakov loop into the system. We find this special boundary condition allows us to probe the nonzero charge sectors of the theory. It is discovered that the energy gap extracted from this special choice of boundary conditions exhibits similar FSS compared to the energy gap extracted from the Polyakov loop. Again, this FSS is found to persist into the continuous-time limit. Finally, in Sec. VI, we give a summary and concluding remarks about the pertinence of this study to the possibility of quantum simulating the twodimensional Abelian Higgs model using a ladder set-up with cold atoms in an optical lattice.

\section{THE ABELIAN HIGGS MODEL AND THE POLYAKOV LOOP}

\section{A. The model and its gauge-invariant reformulation}

In this paper, we consider the compact Abelian Higgs model (compact scalar electrodynamics) with the Higgs mode frozen to unity in $1+1$ Euclidean spacetime dimensions using a lattice discretization. The lattice has spatial and temporal extents $N_{s}$, and $N_{\tau}$, respectively. We used a variety of boundary conditions including periodic (PBC), open boundary conditions $(\mathrm{OBC})$ in space, and more exotic boundary conditions. We will mention which type was used when necessary; however, typically calculations done in the discrete Lagrangian set-up were done with $\mathrm{PBC}$, while calculations done in the continuous-time limit were done with OBC. This model has been introduced before in Ref. [15]. The action for this model is

$$
\begin{aligned}
S= & -\beta_{\mathrm{pl}} \sum_{x} \sum_{\nu<\mu} \operatorname{Re} \operatorname{Tr}\left[U_{x, \mu \nu}\right] \\
& -\kappa \sum_{x} \sum_{\nu=1}^{2}\left[\phi_{x}^{\dagger} U_{x, \nu} \phi_{x+\hat{\nu}}+\phi_{x+\hat{\nu}}^{\dagger} U_{x, \nu}^{\dagger} \phi_{x}\right],
\end{aligned}
$$

with $\quad U_{x, \mu \nu}=e^{i\left(A_{x, \mu}+A_{x+\mu, \nu}-A_{x+\nu, \mu}-A_{x, \nu}\right)}, \quad U_{x, \mu}=e^{i A_{x, \mu}}$, and $\phi_{x}=e^{i \theta_{x}}$. The gauge coupling enters into $\beta_{\mathrm{pl}}=1 / g^{2}$. The coupling $\kappa$ controls the scalar-field hopping between nearest-neighbor sites. The partition function for this model is

$$
Z=\int \mathcal{D}\left[\phi^{\dagger}\right] \mathcal{D}[\phi] \mathcal{D}[U] e^{-S},
$$

with $\mathcal{D}[\phi]=\prod_{x} d \phi_{x}$, and similarly for the gauge field integration.

Because of the compact variables of integration in the original formulation $(\theta$ and $A$ ) the Boltzmann weights can be expanded using Fourier analysis [20]. From these expansions the partition function can be rewritten exactly in a gauge-invariant way by integrating out the $\theta$ and $A$ fields. One is left with only integer fields on the links and plaquettes which can be further simplified to integer fields only living on the plaquettes. The partition function can then be written as

$$
Z=\sum_{\{m\}}\left(\prod_{x, \nu<\mu} I_{m}\left(\beta_{\mathrm{pl}}\right)\right)\left(\prod_{x, \nu} I_{m-m^{\prime}}(2 \kappa)\right) .
$$

In what follows, we always normalize the Bessel functions by their smallest order; i.e., we use the following definitions: $t_{n}(z) \equiv I_{n}(z) / I_{0}(z), t_{n}(0)=\delta_{n, 0}$. For $0<z<$ $\infty$ we have $1=t_{0}(z)>t_{1}(z)>t_{2}(z)>\ldots>0$. In addition, for large $z, t_{n}(z) \simeq 1-n^{2} /(2 z)$, and for small $z$, $t_{n}(z) \simeq z^{n} /\left(2^{n} n !\right)$.

\section{B. The Polyakov loop}

The Polyakov loop, $P$, is a specific instance of the Wilson loop. The latter is defined by closed loops built out of gauge fields, and is gauge invariant. The Polyakov loop is a Wilson loop which wraps around the temporal direction (with PBC) making it noncontractible. The Polyakov loop is an order parameter for confinement/deconfinement transitions in pure gauge theories. In particular, it monitors the center symmetry of the gauge group, and the screening of a static test charge by the gauge field.

The Polyakov loop is related to the free energy induced by the inclusion of a static charge by [21]

$$
\exp [-F / T] \propto\langle P\rangle,
$$

where $F$ is the free energy, and $T$ is the temperature. As defined above, the Polyakov loop has the form

$$
P=\prod_{n=0}^{N_{\tau}-1} U_{x^{*}+n \hat{\tau}, \hat{\tau}}
$$

in the Abelian Higgs model considered here, which is a loop along a single space-slice. With PBC, the insertion of the Polyakov loop into the system forces a scalar current in the opposite direction in order to lower the system energy; however, the cost for the current to run the length of $N_{\tau}$ is 
controlled by the hopping parameter coupling and the length of $N_{\tau}$, and this cost must be overcome for the Polaykov loop expectation value to be nonzero.

We can re-write the Polyakov loop in terms of the gaugeinvariant variables of Sec. II A. Consider the expectation value of the Polyakov loop,

$$
\langle P\rangle=\frac{1}{Z} \int \mathcal{D}\left[\phi^{\dagger}\right] \mathcal{D}[\phi] \mathcal{D}[U]\left(\prod_{n=0}^{N_{\tau}-1} U_{x^{*}+n \hat{\tau}, \hat{\tau}}\right) e^{-S},
$$

with $x^{*}$ a single specific spatial site. Using the expansions from Sec. II A, we pick up new link integrals on the links which contain the additional $U$ variables from the Polyakov loop,

$$
\int \frac{\theta_{x}}{2 \pi} e^{i\left(n-m_{r}+m_{l}+1\right) \theta_{x}}=\delta_{n, m_{r}-m_{l}-1}
$$

Here the subscripts $l$ and $r$ denote the "left" and "right" plaquette quantum numbers, respectively, to the vertical (temporal) link in question. This shifts the difference in $m \mathrm{~s}$ by one at the links which contain the Polyakov loop, but all the other links remain the same. Now we can write the expectation value as

$$
\begin{aligned}
\langle P\rangle= & \frac{1}{Z} \sum_{\{m\}}\left[\prod_{x, \nu<\mu} t_{m}\left(\beta_{\mathrm{pl}}\right)\right]\left[\prod_{x, \nu} t_{m-m^{\prime}}(2 \kappa)\right] \\
& \times\left[\prod_{n=0}^{N_{\tau}-1} \frac{t_{m-m^{\prime}-1}(2 \kappa)}{t_{m-m^{\prime}}(2 \kappa)}\right] .
\end{aligned}
$$

Here the last product is over those links from Eq. (6) that are included in the Polyakov loop. This allows us to identify the Polyakov loop in terms of the new variables as

$$
P=\prod_{n=0}^{N_{\tau}-1} \frac{t_{m-m^{\prime}-1}(2 \kappa)}{t_{m-m^{\prime}}(2 \kappa)}
$$

\section{ISOTROPIC CALCULATIONS OF THE POLYAKOV LOOP}

\section{A. MC and TRG}

In this section, we explore the construction and calculation of the Polyakov loop in terms of the reformulated $m$ variables using the TRG. In order to check our work and results, we compared with traditional MC methods. The $\mathrm{MC}$ algorithms used were the same as those implemented in Ref. [15]. For the TRG calculations we used the higherorder tensor renormalization group (HOTRG) method. We typically used a bond dimension, $D_{\text {bond }}$, of 41 states, and in some cases, 51 states were used to assess the fluctuations. The tensor used in the calculation was constructed in a similar way to that described in Ref. [15]. The main tensor was constructed from a $B$ tensor,

$$
B_{m_{1} m_{2} m_{3} m_{4}}\left(\beta_{\mathrm{pl}}\right)=t_{m_{1}}\left(\beta_{\mathrm{pl}}\right) \delta_{m_{1}, m_{2}} \delta_{m_{2}, m_{3}} \delta_{m_{3}, m_{4}},
$$

(no sum) and the four indices of that tensor were contracted with the square-root, or Cholesky decomposition, of the $A$ tensor,

$$
t_{m-m^{\prime}}(2 \kappa) \equiv A_{m m^{\prime}}(2 \kappa)=L_{m \alpha} L_{\alpha m^{\prime}}^{\dagger}(2 \kappa) .
$$

This is possible when the eigenvalues of this matrix are positive which, in all cases considered here, they are. One can then combine the $L$ matrix here with the $B$ tensor from Ref. [15]. The transfer matrix can be constructed by blocking along a time-slice with the tensor described above.

To find the expectation value of the Polaykov loop, we considered another transfer matrix, which possesses an impure temporal link: that link which is shifted by one due to the addition of the line of $U \mathrm{~s}$ from the Polyakov loop. This can be accomplished by inserting a matrix into the end of the blocked time-slice such that when the ends are closed (due to PBC) the resulting $A$ matrix which would be completed by this contraction is instead a matrix of the form,

$$
t_{m-m^{\prime}-1}(2 \kappa) \equiv \tilde{A}_{m m^{\prime}}(2 \kappa)
$$

Since this is a transfer matrix, this impure matrix will appear $N_{\tau}$ times in the product thus defining the Polyakov loop of length $N_{\tau}$.

A comparison between the HOTRG method and MC for computing the Polyakov loop can be seen in Fig. 1. In this figure, we keep a fixed spatial and temporal extent with $\mathrm{PBC}$, and varied the gauge coupling along a fixed range of $\kappa$ values. This can be compared with calculations done in Ref. [22]. Another example of the comparison between the two can be

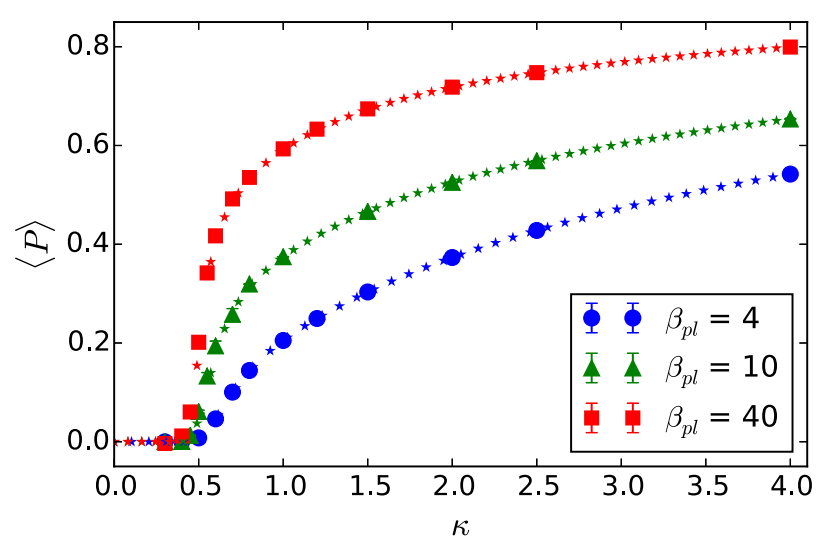

FIG. 1. Comparison between HOTRG and MC for a range of $\kappa$ and $\beta_{\mathrm{pl}}$ values. Here the HOTRG data appear as the star markers, while the MC appears as the square, triangle, and circle markers with error-bars. This was done on a lattice of size $N_{s}=N_{\tau}=16$. 
TABLE I. A table comparing MC and HOTRG values for the Polyakov loop. The upper values in each cell are the values gotten from HOTRG blocking, while the lower values are those from MC calculations and their jack-knife errors. These were generated at $\beta_{\mathrm{pl}}=5$ with $N_{s}=16$. Here the number of states kept by the tensor truncation was $D_{\text {bond }}=41$, and $D_{\text {bond }}=33$ was used to estimate the errors.

\begin{tabular}{lcccc}
\hline \hline & $\kappa=0.5$ & $\kappa=1$ & $\kappa=1.5$ & $\kappa=2$ \\
\hline$N_{\tau}=16$ & $0.0136(1)$ & $0.2451(1)$ & $0.3424(1)$ & $0.4102(1)$ \\
& $0.013(1)$ & $0.2461(6)$ & $0.3438(5)$ & $0.4102(7)$ \\
$N_{\tau}=32$ & $1.84(2) \mathrm{e}-4$ & $0.0601(1)$ & $0.11725(4)$ & $0.16828(3)$ \\
& $2(4) \mathrm{e}-4$ & $0.0606(4)$ & $0.1175(4)$ & $0.1687(4)$ \\
$N_{\tau}=64$ & $3.4(1) \mathrm{e}-8$ & $0.00361(1)$ & $0.01374(1)$ & $0.02832(2)$ \\
& $-1(3) \mathrm{e}-4$ & $0.0037(4)$ & $0.0137(3)$ & $0.0288(4)$ \\
$N_{\tau}=128$ & $1.15(4) \mathrm{e}-15$ & $1.3(1) \mathrm{e}-5$ & $1.89(1) \mathrm{e}-4$ & $8.02(1) \mathrm{e}-4$ \\
& $3(3) \mathrm{e}-4$ & $4(2) \mathrm{e}-4$ & $3(2) \mathrm{e}-4$ & $4(2) \mathrm{e}-4$ \\
\hline \hline
\end{tabular}

seen in Table I, where we varied the temporal extent of the lattice at fixed spatial size for a variety of $\kappa$ values. These data were a catalyst for the study of the scaling of the free energy, or energy gap, of a static charge inserted into the system via the Polyakov loop. Figure 2 shows the data from Table I of the comparison between MC and HOTRG when varying $N_{\tau}$. Again, this was done using PBC. Overall we find good agreement between the two methods.

\section{B. The energy gap}

Consider the Polyakov loop in terms of the ratio of two partition functions: one with the inclusion of the static charge, and the other without,

$$
\langle P\rangle=\frac{\tilde{Z}}{Z} .
$$

In practice, say for MC calculations, one can sum over the possible locations of $x^{*}$ in Eq. (6) and divide by $N_{s}$. Due to

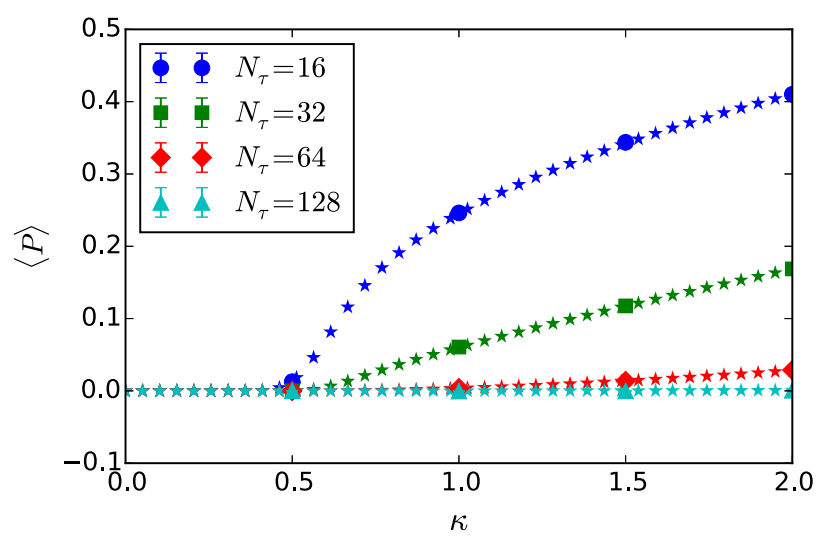

FIG. 2. HOTRG and MC data with fixed spatial length and various temporal lengths. Data like this were used to find the decay in the temporal direction of the lattice. Here the stars are the HOTRG data while the squares, circles, diamonds, and triangles are the MC data with error bars. This was done on a $N_{s}=16$ lattice with $\beta_{\mathrm{pl}}=5$, and $D_{\text {bond }}=41$. translation invariance one just recovers $N_{s}$ copies of the same number. By re-writing the partition function as a trace over products of transfer matrices, we can expose the dependence on $N_{\tau}$,

$$
\langle P\rangle=\frac{\tilde{Z}}{Z}=\frac{\operatorname{Tr}\left[\tilde{\mathbb{U}}^{N_{\tau}}\right]}{\operatorname{Tr}\left[\mathbb{T}^{N_{\tau}}\right]}=\frac{\sum_{i=0}^{N} \tilde{\lambda}_{i}^{N_{\tau}}}{\sum_{i=0}^{N} \lambda_{i}^{N_{\tau}}},
$$

where in the last step we have diagonalized the transfer matrices. This makes it clear that in the large $N_{\tau}$ limit the Polyakov loop expectation value is dominated by the largest eigenvalues $\tilde{\lambda}_{0}$ and $\lambda_{0}$. Thus, we find,

$$
\begin{aligned}
\log \langle P\rangle & \simeq N_{\tau} \log \left(\tilde{\lambda}_{0} / \lambda_{0}\right) \\
& =-N_{\tau} \Delta E,
\end{aligned}
$$

with $\Delta E$ the energy gap between the ground state of the system with the static charge, and that without, for sufficiently large $N_{\tau}$. From the previous steps, we see that at sufficiently large $N_{\tau}$ (or low temperatures),

$$
\langle P\rangle \simeq e^{-N_{\tau} \Delta E} .
$$

This relationship is clear in Fig. 3, where the linear behavior is seen on a log-plot. In addition, the $y$-intercept is approximately zero, indicating $N_{\tau}$ has been taken large enough. To further test the agreement between $\mathrm{MC}$ and HOTRG, we compared the energy gap values for a few spatial lattice sizes at fixed $\kappa$ using PBC. Fig. 4 demonstrates the agreement for $\Delta E$ values.

\section{Universality: Expectations and conjectures}

For large enough $\kappa$, i.e., greater than the BerezinskiiKosterlitzThouless (BKT) transition value, and small enough $g^{2} N_{s}$, we expect that

$$
\Delta E \simeq \frac{a}{N_{s}}+b g^{2} N_{s},
$$




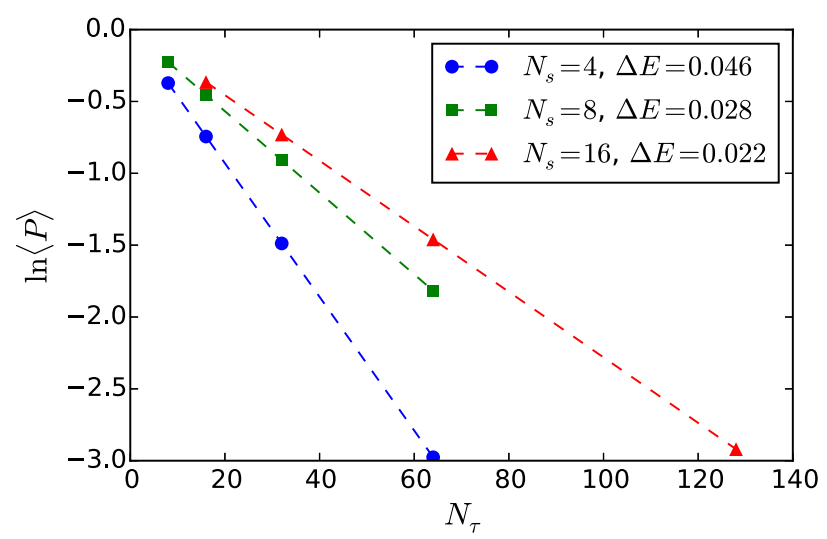

FIG. 3. The energy gap for different spatial sizes with $\kappa=1.6$ and $\beta_{\mathrm{pl}}=44$. In general, the slope depends on $N_{s}, \beta_{\mathrm{pl}}$, and $\kappa$. The dashed lines are not fits, merely lines connected between dots to guide the eye. $D_{\text {bond }}=41$ was used for these HOTRG calculations.

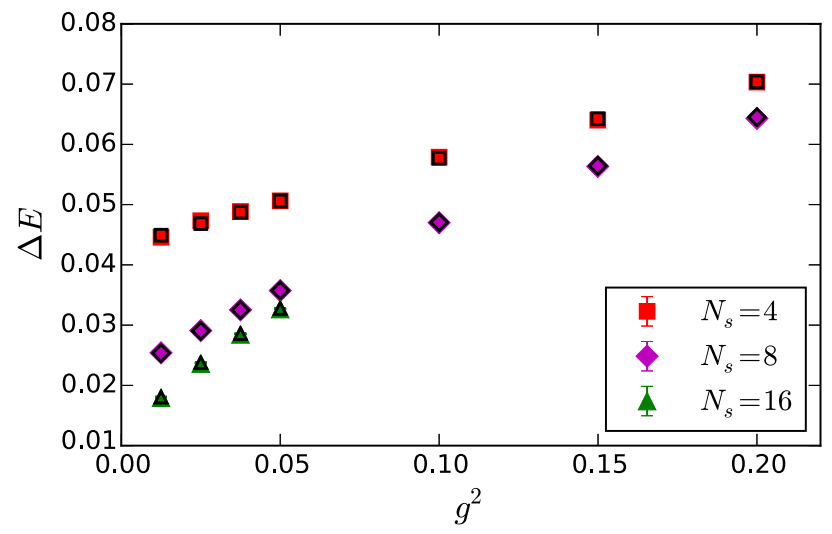

FIG. 4. This figure shows a comparison between $\mathrm{MC}$ and HOTRG for values of $\Delta E$ calculated at a fixed value of $\kappa=1.6$. Here $D_{\text {bond }}=41$ was used for the tensor truncation. The solid markers are the MC data, while the black empty markers are the HOTRG data.

where $a$ and $b$ are still functions of $\kappa$. In the limit where $g^{2}$ becomes zero, this is just the statement that $\Delta E$ goes to zero in the limit of large $N_{s}$, a consequence of the gapless BKT phase at infinite volume. The guessed correction corresponds to a linear potential. If we multiply Eq. (18) by $N_{s}$, then the right-hand side depends only on $g^{2} N^{2}$. We conjecture that this feature persists beyond the lowest order approximation, namely,

$$
\Delta E N_{s}=f\left(g^{2} N_{s}^{2}\right)
$$

Figure 5 supports this idea and shows a reasonably good data collapse across a wide range. In addition, we can observe that for larger $g^{2} N_{s}^{2}, f\left(g^{2} N_{s}^{2}\right) \sim g N_{s}$, which means that in this intermediate regime, $\Delta E$ become approximately independent

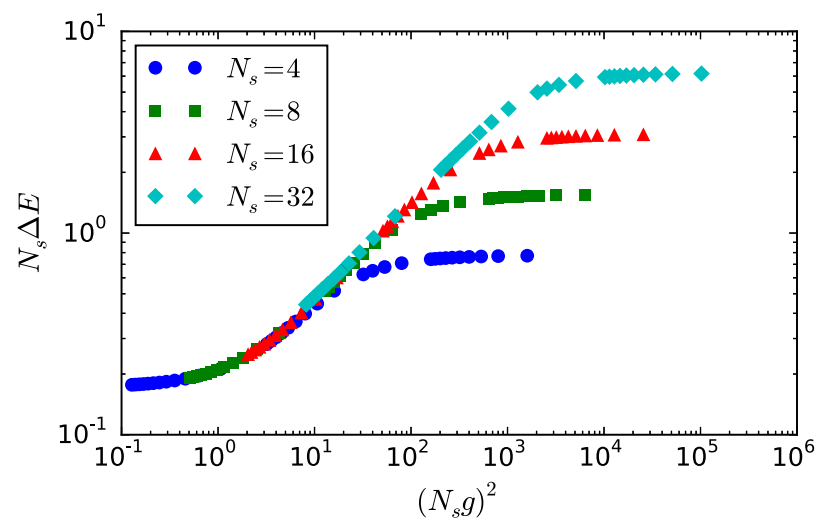

FIG. 5. Data collapse across different $N_{s}$ for sufficiently small $g$, and collapse breaking across different $N_{s}$ at large $g$ in the case of isotropic coupling. Here $\kappa=1.6$, and $D_{\text {bond }}=41$ was used in the HOTRG calculations.

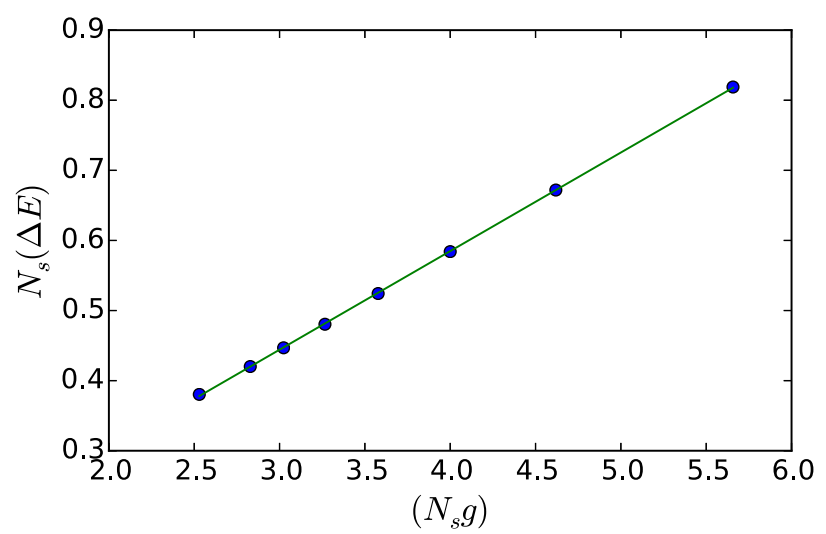

FIG. 6. Data from the collapse data set. Here the intermediate region is plotted which exhibits a $N_{s} \Delta E \propto N_{s} g$ behavior meaning the energy gap is proportional to the gauge coupling. The solid line is a linear fit through the data (dots).

of $N_{s}$ and is proportional to $g$. This intermediate region is shown in Fig. 6 where the proportionality between $\Delta E$ and $g$ is clear, and the data are plotted against a linear fit.

However, the data collapse breaks down if we increase $g$ to large values while keeping $N_{s}$ constant. For very large $g$ (small $\beta_{\mathrm{pl}}$ ), the lowest energy state corresponds to having all plaquette quantum numbers set to zero. This is accomplished when the matter loop follows exactly the Polyakov loop in the opposite direction. This state contributes $\left(t_{1}(2 \kappa)\right)^{N_{\tau}}$ to the partition function with a corresponding energy difference, $\Delta E$, of $-\ln \left(t_{1}(2 \kappa)\right)$ in the large $N_{\tau}$ limit. Thus, for large values of $g$, we expect

$$
\Delta E \rightarrow-\ln \left(t_{1}(2 \kappa)\right)
$$

independent of $N_{s}$. 


\section{THE POLYAKOV LOOP IN THE CONTINUOUS-TIME LIMIT}

\section{A. The spin-1 Hamiltonian}

In Ref. [15], the continuous-time limit for the Abelian Higgs model was taken in the field quantum number representation in the limit the Higgs quartic self-coupling is infinitely large. We summarize the important points here. To take the continuous-time limit, one takes $\kappa_{\tau}, \beta_{\mathrm{pl}} \rightarrow \infty$ while simultaneously taking $\kappa_{s}$, and the temporal lattice spacing, $a$, to zero such that the combinations

$$
U \equiv \frac{1}{\beta_{\mathrm{pl}} a}=\frac{g^{2}}{a}, \quad Y \equiv \frac{1}{2 \kappa_{\tau} a}, \quad X \equiv \frac{2 \kappa_{s}}{a},
$$

are finite. Note that $X$ here is related to $\tilde{X}$ in Ref. [15] by $X=\sqrt{2} \tilde{X}$. In this limit, and using the properties of the $t_{n}(z)$ functions mentioned in Sec. II A, the transfer matrix is close to the identity. Keeping to first-order in each coupling constant, a Hamiltonian with a three-state, or spin-1, approximation can be identified as

$H=\frac{U}{2} \sum_{i=1}^{N_{s}}\left(L_{i}^{z}\right)^{2}+\frac{Y}{2} \sum_{i}^{\prime}\left(L_{i+1}^{z}-L_{i}^{z}\right)^{2}-\frac{X}{\sqrt{2}} \sum_{i=1}^{N_{s}} L_{i}^{x}$,

where the sum, $\sum_{i}{ }^{\prime}$, takes the OBC into account and includes $\left(L_{1}^{z}\right)^{2}+\left(L_{N_{s}}^{z}\right)^{2}$. This Hamiltonian describes a three-state system with a local Hilbert state-space of $m= \pm 1,0$.

The first term represents the plaquette interactions. The second term is associated with the integration of the time links. They can be interpreted as charges determined by Gauss's law, in other words the difference between the two plaquettes (electric field) on each side of the link. Finally, the third term represents the spatial hopping term and reveals the equivalence between the spatial hopping of charge and the local spin-flip of the electric field.

\section{B. The spin- $n$ Hamiltonian}

In order to extend and improve the study of this model in the continuous-time limit, it is advantageous to consider a spin representation of the $L^{z}$ operator greater than one. The $X$ term in Eq. (21) must be modified then, since, higher spin representations of the $L^{x}$ operator do not accurately represent the genuine continuous-time limit of the Abelian Higgs model.

The operator $U^{x}=\frac{1}{2}\left(U^{+}+U^{-}\right)$, with $U^{+}$and $U^{-}$as special types of raising and lowering operators for field quantum numbers, is the appropriate replacement for $L^{x}$ (the notation $U^{x}$ is firstly not to be confused with the parameter $U$ which parameterizes the strength of the gauge field interaction, and secondly is used to indicate that the action of $U^{x}$ on a state is similar in spirit to $L^{x}$; however, with different matrix elements). Note that $U^{ \pm}$are different from the ladder operators in the angular momentum algebra. In the basis of the eigenvectors of $L^{z}$, applying $U^{+}\left(U^{-}\right)$to them also raises (lowers) the electric field quantum number by 1 but with all coefficients 1 ,

$$
U^{ \pm}|m\rangle=|m \pm 1\rangle .
$$

The action of $L^{z}$ is the same, $L^{z}|m\rangle=m|m\rangle$. Now the quantum Hamiltonian for the Abelian Higgs model for arbitrary spin is written,

$H=\frac{U}{2} \sum_{i=1}^{N_{s}}\left(L_{i}^{z}\right)^{2}+\frac{Y}{2} \sum_{i}^{\prime}\left(L_{i+1}^{z}-L_{i}^{z}\right)^{2}-X \sum_{i=1}^{N_{s}} U_{i}^{x}$,

where $X$ is the same as in Eq. (22) because of the definition of $U^{x}$. We will still call the $2 n+1$ state truncation a "spin- $n$ " truncation for convenience, even though we no longer are using the angular momentum algebra.

\section{The charge representation}

By Gauss's Law, the charge (link) quantum numbers are defined as $\bar{L}_{i+\frac{1}{2}}^{z}=L_{i+1}^{z}-L_{i}^{z}$. This allows one to use the charge representation. The corresponding $\bar{U}^{ \pm}$have the same form. If we increase the field (plaquette) quantum number at site $i$ by 1 , the charge quantum number $\bar{L}_{i+\frac{1}{2}}^{z}$ will be decreased by 1 , but $\bar{L}_{i-\frac{1}{2}}^{z}$ will be increased by 1 . So the ladder operators in the field representation are related with the ones in charge representation by

$$
U_{i}^{+}=\bar{U}_{i-\frac{1}{2}}^{+} \bar{U}_{i+\frac{1}{2}}^{-} \text {. }
$$

With $\mathrm{OBC}, \bar{L}_{\frac{1}{2}}^{z}=L_{1}^{z}, \bar{L}_{N_{s}+\frac{1}{2}}^{z}=-L_{N_{s}}^{z}$, and we can solve for field quantum numbers in terms of charge quantum numbers

$$
L_{i}^{z}=\sum_{j=0}^{i-1} \bar{L}_{j+\frac{1}{2}}^{z}
$$

Then Eq. (24) reads

$$
\begin{aligned}
\bar{H}= & \frac{U}{2} \sum_{0 \leq j, k<N_{s}} c_{j k} \bar{L}_{j+\frac{1}{2}}^{z} \bar{L}_{k+\frac{1}{2}}^{z}+\frac{Y}{2} \sum_{i=0}^{N_{s}}\left(\bar{L}_{i+\frac{1}{2}}^{z}\right)^{2} \\
& -\frac{X}{2} \sum_{i=1}^{N_{s}}\left(\bar{U}_{i-\frac{1}{2}}^{+} \bar{U}_{i+\frac{1}{2}}^{-}+\bar{U}_{i-\frac{1}{2}}^{-} \bar{U}_{i+\frac{1}{2}}^{+}\right),
\end{aligned}
$$

where $c_{j k}=N_{s}-\max \{j, k\}$. Note that Hamiltonian (24) has $N_{s}$ sites, while there are $N_{s}+1$ sites in Hamiltonian (27). The former has total charge zero, while the latter 
has charge conservation symmetry and can represent all charge sectors. Although Hamiltonian (27) has long range interactions in the first term, a similar form has been used to study the real-time dynamics of the Schwinger model with qubits by coarse graining in time [2]. In our case, we focus on Hamiltonian (24) as it has only nearest-neighbor interactions which allows easy implementations on optical lattices.

In the following, we used the density matrix renormalization group (DMRG) $[23,24]$ for our studies in the continuous-time limit. The finite DMRG algorithm with matrix-product-state (MPS) [25] optimization was performed using the ITensor $\mathrm{C}++$ library [26]. Note that the quantum entanglement comes from the nearestneighbor interaction in the field representation, while it comes from the nearest-neighbor hopping in the charge representation. In the gapless phase where $X$ is relatively larger than $Y$, the bipartite entanglement entropy for the field representation is much smaller than that of the charge representation, so a much smaller bond dimension is needed for the former case using MPS.

\section{D. $H$ with the Polyakov loop}

Using the reformulation from Sec. II B, we can follow the same prescription for taking the continuous-time limit from Sec. IVA and apply it to the Polaykov loop. This implies taking the same limit for the $P$ operator. We find,

$$
P \rightarrow 1+\frac{1}{2\left(2 \kappa_{\tau}\right)}\left(2\left(m-m^{\prime}\right)-1\right)+\mathcal{O}\left(\left(2 \kappa_{\tau}\right)^{-2}\right)
$$

This corresponds to an additional term in the quantum Hamiltonian which is located at a single specific site, and allows us to write,

$$
\tilde{H}=H-\frac{Y}{2}\left(2\left(L_{i^{*}+1}^{z}-L_{i^{*}}^{z}\right)-1\right)
$$

where $\tilde{H}$ is the quantum Hamiltonian corresponding to the addition of a static charge at a specific site, $i^{*}$, and $H$ is the original quantum Hamiltonian of the Abelian Higgs model from Eq. (24).

After taking this limit, it's clear that the energy gap, $\Delta E$, between the ground states of $\tilde{H}$ and $H$ is due to the additional term and the inserted static charge. This energy gap, when calculated in the low-temperature limit, is the same quantity that is calculated in the isotropic coupling case at large $N_{\tau}$ from Sec. III B.

In numerical calculations, and to avoid the effects of boundary conditions as much as possible, we put the Polyakov loop on the middle link of the square lattice (in the isotropic coupling picture), or equivalently, we remove the $\left(N_{s} / 2\right)$ th term from the $Y$ sum in Hamiltonian

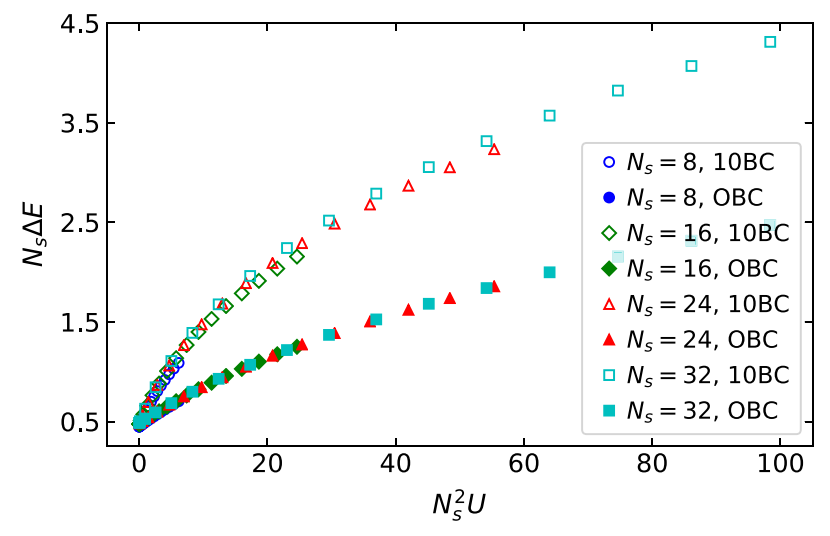

FIG. 7. Data collapse of $N_{s} \Delta E$ as a function of $N_{s}^{2} U$ with $X=2$. The upper curve (open markers) is the data collapse in the case of an external electric field with no Polyakov loop (see Sec. V B), and the lower curve (solid markers) is the case of OBC with a Polaykov loop included. These calculations were done using the DMRG with MPS.

(24) and shift it by one. Then the Hamiltonian with the Polyakov loop included reads

$$
\begin{aligned}
\tilde{H}= & \frac{U}{2} \sum_{i=1}^{N_{s}}\left(L_{i}^{z}\right)^{2}+\frac{Y}{2} \sum_{i \neq \frac{N_{s}}{2}}^{\prime}\left(L_{i+1}^{z}-L_{i}^{z}\right)^{2} \\
& +\frac{Y}{2}\left(L_{\frac{N_{s}}{2}+1}^{z}-L_{\frac{N_{s}}{2}}^{z}-1\right)^{2}-X \sum_{i=1}^{N_{s}} U_{i}^{x} .
\end{aligned}
$$

By a simple rearranging of terms this can be cast into the form from Eq. (29). So adding a Polyakov loop creates a single charge in the neutral system [i.e., Eq. (24)].

Just as in the isotropic-coupling picture, the continuoustime limit preserves the collapse of $\Delta E$ across a range of couplings and spatial sizes [17]. A glance at Eq. (14) shows that in the continuous-time limit $\Delta E$ is the difference between the ground state energy eigenvalues of a system with the Polyakov loop included, and one without it. This can be readily calculated from the DMRG, and the collapse can be seen in Fig. 7. Even more remarkable is that in an appropriate regime, up to a rescaling by $\kappa$, the $\Delta E$ calculated in the continuous-time limit is equal to the $\Delta E$ calculated in the isotropic-coupling discrete case. Further details about this can be found in Ref. [17] where this relationship is proposed as an observable seen in quantum simulations of this model.

\section{E. The $\mathbf{O ( 2 )}$ limit}

In our previous work, we discussed the $\mathrm{O}(2)$ limit where $U \rightarrow 0\left(g^{2} \rightarrow 0\right)$, and the $\kappa_{\tau} \gg \beta_{\mathrm{pl}} \gg \kappa_{s}$ limit separately, where we used the charge representation and field representation with a three state truncation respectively. Because the $\mathrm{O}(2)$ model has a gapless phase where high quantum number states are easily excited, a three-state truncation is 


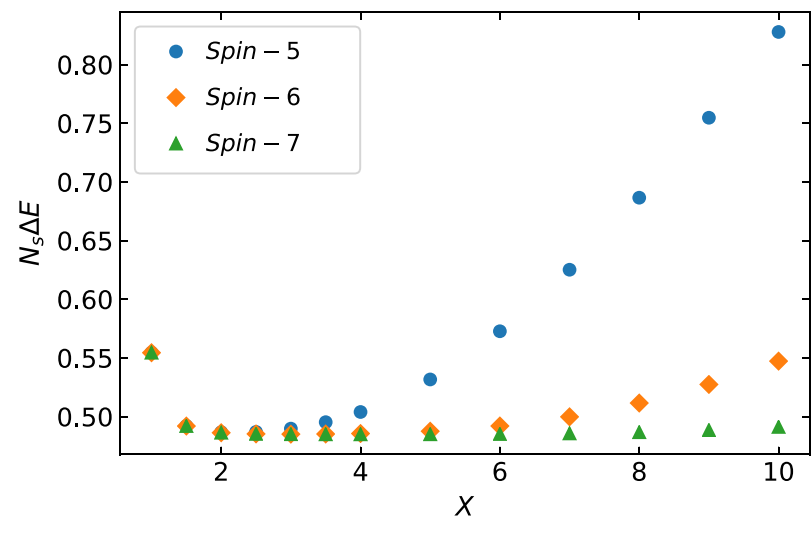

FIG. 8. The enegy gap in the $\mathrm{O}(2)$ limit with $N_{s}=32$ in field representation as a function of $X$. These calculations were done using the DMRG with MPS.

far from enough for Hamiltonian (24). Here we want to connect these two limits continuously by keeping more states in the truncation.

To test how many states we should keep to simulate the $\mathrm{O}(2)$ model using Eq. (24) with $U=0$, we gradually increase the number of states and calculate the energy gap between the ground states of Hamiltonians (24) and (30). In these calculations, we used units of $Y=1$ in the Hamiltonian. Studying the data collapse requires precise values of $N_{s} \Delta E$ in the $\mathrm{O}(2)$ limit. The energy gap of the $\mathrm{O}(2)$ model in the BKT region scales like $1 / N_{s}$ in a polynomial form, with the coefficient of $1 / N_{s}$ constant, which is verified in Fig. 8 where $N_{s} \Delta E$ is almost constant for a large range of $X \in[2,10]$ with $N_{s}=32$. We also see that a spin-5 truncation is good enough for $X \approx 2$, and a spin-6 truncation is essentially perfect for $X \in[2,4]$.

Finite-size effects also play a role. As shown in Fig. 9, at $X=2$, a spin-5 truncation breaks down after $N_{s}=40$, but a spin-6 truncation works perfectly well for $N_{s} \leq 64$. The extrapolation of $N_{s} \Delta E$ to the thermodynamic limit gives

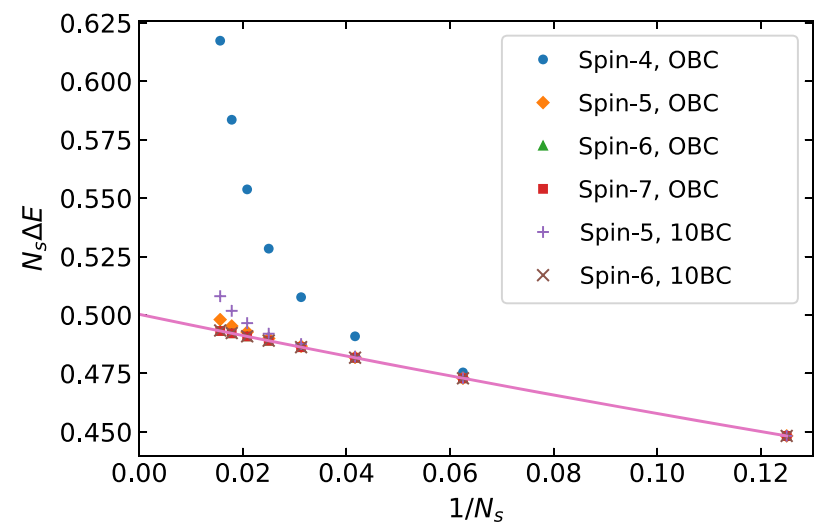

FIG. 9. The enegy gap in the $\mathrm{O}(2)$ limit with $X=2$ in plaquette quantum number representation as a function of $1 / N_{s}$ for different spin-truncations. These calculations were done using the DMRG with MPS.

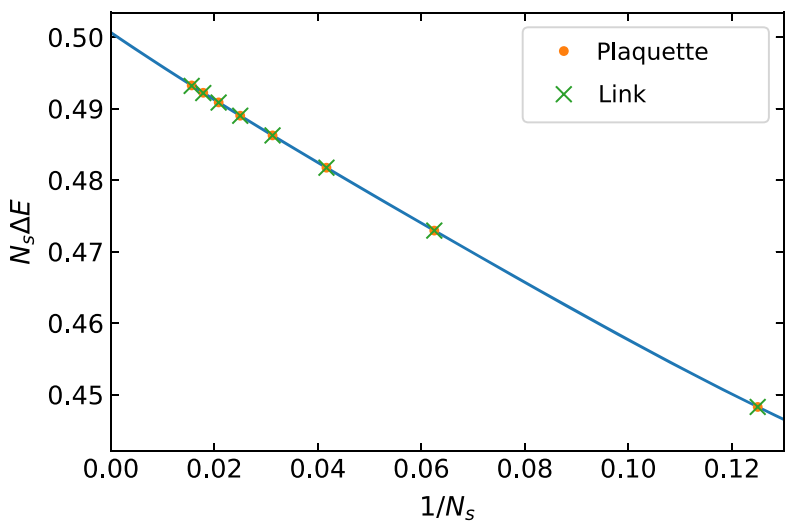

FIG. 10. The enegy gap in the $\mathrm{O}(2)$ limit with a spin-6 truncation in the plaquette quantum number representation and link quantum number representation as a function of $1 / N_{s}$. Here $N_{s}=8,16, \ldots, 64$ with $X=2$ fixed. These calculations were done with the DMRG using MPS.

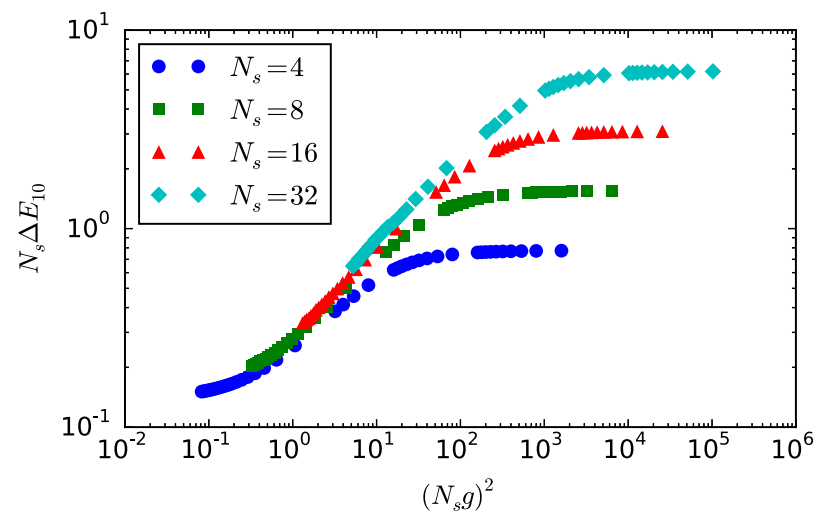

FIG. 11. The energy gap between the 10BC partition function and the $\mathrm{OBC}$ partition function in the case of isotropic coupling. This is for $\kappa=1.6$ and $D_{\text {bond }}=41$ for the HOTRG truncation. Similar to the Polyakov loop gap, for sufficiently small $g$, we see data collapse, and for large enough $g$, we see the collapse breakdown.

$N_{s} \Delta E=0.50029(8)$, which is consistent with $Y / 2=0.5$ in units of $Y$ in the continuous-time limit. By calculating the energy gap between the charge- 0 and charge- 1 sectors with Hamiltonian (27), we show that the energy gaps for the two representations of $\mathrm{O}(2)$ converge to the same values with a spin-6 truncation. This can be seen in Fig. 10.

\section{REPLACING THE POLYAKOV LOOP BY SPECIAL BOUNDARY CONDITIONS}

\section{A. Isotropic coupling}

By inserting the Polyakov loop, one probes the response of the system to the addition of a single static charge. For a total charge, $Q$, the $Q \neq 0$ sectors of the theory can be probed by changing the boundary conditions of the system, this is like subjecting the system to an external electric 
field. One can consider the pure Abelian Higgs model with a boundary of zeros on one side, and a boundary of ones on the other in the field-quantum-number representation. With the conventions used in this text, to put the system in the $Q=-1$ sector amounts to setting a boundary of ones on the left side of the system, and a boundary of zeros on the right side (10BC). With these boundary conditions (and the now absent Polyakov loop) Gauss's law tells us that there is a total charge of -1 across a time slice. In the tensor language, the boundary tensors, in this case the $B$ tensors, are assigned the state $m=1$ on one side, and $m=0$ on the other.

The relationship between the situation with the Polyakov loop inserted, and the system with special boundary conditions can be made more clear by a simple example. It consists of sliding the Polyakov loop all the way to the boundary of a system with OBC. We will relate the $Q=-1$ sector (which is identical to the $Q=1$ sector) to the Polyakov loop. Take a system where, along a time slice, charge is defined at a link as the difference of the electric field as $n=m_{\text {right }}-m_{\text {left }}$, with $Q=\sum n$. Then at the link with the Polyakov loop one has $n=m_{\text {right }}-m_{\text {left }}-1$. If one slides this all the way to the left-most boundary link, it becomes $n=m_{\text {right }}-0-1=m_{\text {right }}-1$. This is precisely the charge one would find by setting the left boundary quantum numbers to one and removing the Polaykov loop entirely. By Gauss's law the charge of this system would be -1 . Thus, a system with skewed boundary conditions of zeros on one side and ones on the other is equal to a system with OBC with a Polaykov loop pushed to the boundary link.

Since the Polyakov loop is related to a special circumstance of boundary conditions, we would expect the energy gap from the inclusion of the Polyakov loop to be qualitatively similar to the energy gap between the $Q=1$ and $Q=0$ sectors. Indeed, a collapse can be found for the energy gap between the 10BC system, and that with OBC. This is shown in Fig. 11. Again, there is collapse for sufficiently small $g$, and collapse breaking at large enough $g$. In addition, $\Delta E_{10} \rightarrow-\ln \left(t_{1}(2 \kappa)\right)$ for large $g$ regardless of $N_{s}$, which is in agreement with the Polyakov loop result.

\section{B. Continuous-time limit}

Similar to the isotropic coupling case, in the continuoustime limit special boundary conditions can be imposed to probe the $Q \neq 0$ sectors of the theory. Whereas before, in Secs. IV B and IV D, OBC were used (zeros on both spatial ends) and the Polyakov loop was inserted in the center, one can consider the pure model in the absence of the Polyakov loop, and change the left spatial-boundary end-point to one. By Gauss's law, one can see that this leaves the system with total charge $Q=-1$.

To implement these boundary conditions in DMRG, one has to imagine there are two additional sites on the chain, one to the left, and one to the right. The right-side site, say, has quantum number zero (the same as in the typical OBC case), however on the left-side site we assign value one. To include the effect of the boundary conditions we rewrite the Hamiltonian accordingly:

$$
\begin{aligned}
H_{10}= & \frac{U}{2} \sum_{i=1}^{N_{s}}\left(L_{i}^{z}\right)^{2}+\frac{Y}{2} \sum_{i=1}^{N_{s}-1}\left(L_{i+1}^{z}-L_{i}^{z}\right)^{2} \\
& +\frac{Y}{2}\left(L_{N_{s}}^{z}\right)^{2}+\frac{Y}{2}\left(L_{1}^{z}-1\right)^{2}-X \sum_{i=1}^{N_{s}} U_{i}^{x} .
\end{aligned}
$$

This is the form of the Hamiltonian in the $Q=-1$ sector, in contrast to (24) which is the Hamiltonian with zeros on the boundaries (the $Q=0$ sector). In Fig. 7, one can see the data collapse of the energy gap between systems with 10BC and OBC across a range of spatial sizes and gauge couplings. An understanding of the relationship between the $Q=-1$ Hamiltonian, and the Polyakov loop Hamiltonian can be understood through a change of variables. This is worked out in Ref. [17] where the two systems are related by a linear potential term.

\section{CONCLUSIONS}

In this paper, we have studied the expectation value of the Polyakov loop in the compact Abelian Higgs model in $1+1$ dimensions. In our study, we compared the TRG, $\mathrm{MC}$, and the DMRG method and found excellent agreement between them. It was found that the Polaykov loop is related to the energy gap between charge-sectors of the Abelian Higgs model and that this energy gap exhibits universal finite-size scaling behavior. The scaling of the energy gap was studied in both the fully discrete lattice system and the continuous-time quantum limit of this model, and it was found that the universal behavior of the energy gap survives this limit. In addition, special boundary conditions were able to reproduce similar features of the data collapse found from the Polyakov loop which provides an alternate method to study this energy gap.

The results in this paper give support to Ref. [17] where it is proposed to use the two-dimensional Abelian Higgs model as a proof of principle model for the case of quantum simulation using cold atoms in optical lattices. In that reference, the energy gap associated with the Polyakov loop and its FSS collapse are suggested as observables for the simulation. This work shows, in detail, the passage from the discrete Lagrangian lattice model to the continuoustime quantum Hamiltonian model. This is done in a way such that gauge invariance is maintained exactly and the result is suitable for quantum simulation. That is, configurations in the quantum simulation are those of the physical state-space of the model (up to spin truncations). 
An interesting feature found in our study is that at finite space-time volume and weak gauge coupling, there is a sudden increase of the Polyakov loop when we increase $\kappa$ beyond the critical value corresponding to the BKT transition. Since the primary scope of this work is to explore results at finite volume and finite lattice spacing in order to move the prospect of quantum simulating gauge theories using cold atoms in optical lattices forward, understanding this sudden increase in the context of the continuum limit and the infinite volume limit is left for future work.

\section{ACKNOWLEDGMENTS}

We thank J. Zeiher for valuable conversations. Part of this work was done while Y. M. visited the GGI in Florence during the workshop "From Static to Dynamical Gauge Fields with Ultracold Atoms." This work was supported in part by the U.S. Department of Energy (DOE) under Awards No. DE-SC0010113 (Y.M.) and No. DESC0009998 (J. U. Y.) and by the National Science Foundation under Grant No. DMR-1411345 (S. W. T.). J. Z. and J. U. Y. contributed equally to this work.
[1] S. Coleman, Aspects of Symmetry (Cambridge University Press, Cambridge, England, 1985).

[2] E. A. Martinez, C. A. Muschik, P. Schindler, D. Nigg, A. Erhard, M. Heyl, P. Hauke, M. Dalmonte, T. Monz, P. Zoller, and R. Blatt, Nature (London) 534, 516 (2016).

[3] D. Banerjee, M. Dalmonte, M. Müller, E. Rico, P. Stebler, U.-J. Wiese, and P. Zoller, Phys. Rev. Lett. 109, 175302 (2012).

[4] V. Kasper, F. Hebenstreit, F. Jendrzejewski, M. K. Oberthaler, and J. Berges, New J. Phys. 19, 023030 (2017).

[5] N. Klco, E. F. Dumitrescu, A. J. McCaskey, T. D. Morris, R. C. Pooser, M. Sanz, E. Solano, P. Lougovski, and M. J. Savage, Phys. Rev. A 98, 032331 (2018).

[6] Y. Kuno, K. Kasamatsu, Y. Takahashi, I. Ichinose, and T. Matsui, New J. Phys. 17, 063005 (2015).

[7] Y. Kuno, S. Sakane, K. Kasamatsu, I. Ichinose, and T. Matsui, Phys. Rev. D 95, 094507 (2017).

[8] D. Gonzlez-Cuadra, E. Zohar, and J. I. Cirac, New J. Phys. 19, 063038 (2017).

[9] Z. Y. Xie, J. Chen, M. P. Qin, J. W. Zhu, L. P. Yang, and T. Xiang, Phys. Rev. B 86, 045139 (2012).

[10] Y. Meurice, Phys. Rev. B 87, 064422 (2013).

[11] Y. Liu, Y. Meurice, M. P. Qin, J. Unmuth-Yockey, T. Xiang, Z. Y. Xie, J. F. Yu, and H. Zou, Phys. Rev. D 88, 056005 (2013).

[12] A. Denbleyker, Y. Liu, Y. Meurice, M. P. Qin, T. Xiang, Z. Y. Xie, J. F. Yu, and H. Zou, Phys. Rev. D 89, 016008 (2014).
[13] J. F. Yu, Z. Y. Xie, Y. Meurice, Y. Liu, A. Denbleyker, H. Zou, M. P. Qin, J. Chen, and T. Xiang, Phys. Rev. E 89, 013308 (2014).

[14] C. Gattringer, T. Kloiber, and M. Müller-Preussker, Phys. Rev. D 92, 114508 (2015).

[15] A. Bazavov, Y. Meurice, S.-W. Tsai, J. Unmuth-Yockey, and J. Zhang, Phys. Rev. D 92, 076003 (2015).

[16] J. Zeiher, R. van Bijnen, P. Schauß, S. Hild, J.-y. Choi, T. Pohl, I. Bloch, and C. Gross, Nat. Phys. 12, 1095 (2016).

[17] J. Zhang, J. Unmuth-Yockey, A. Bazavov, S. W. Tsai, and Y. Meurice, arXiv:1803.11166.

[18] E. Zohar, J. I. Cirac, and B. Reznik, Rep. Prog. Phys. 79, 014401 (2016).

[19] B. Gadway, talk given at the NPQI Meeting, Argonne, 2018.

[20] R. Savit, Rev. Mod. Phys. 52, 453 (1980).

[21] I. Montvay and G. Munster, Quantum Fields on a Lattice, Cambridge Monographs on Mathematical Physics (Cambridge University Press, Cambridge, England, 1997).

[22] E. Poppitz and Y. Shang, Int. J. Mod. Phys. A 23, 4545 (2008).

[23] S. R. White, Phys. Rev. Lett. 69, 2863 (1992).

[24] U. Schollwck, Ann. Phys. (Amsterdam) 326, 96 (2011), Special Issue.

[25] S. Östlund and S. Rommer, Phys. Rev. Lett. 75, 3537 (1995).

[26] Version 2.1.1, http://itensor.org/. 